\section{Check for updates}

Cite this: Nanoscale, 2021, 13, 8740

\title{
Cell-free exosome-laden scaffolds for tissue repair
}

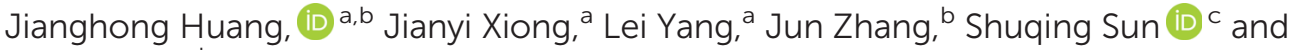 \\ Yujie Liang*d
}

\begin{abstract}
With the development of regenerative medicine, tissue repair at the molecular, cellular, tissue, and organ level has seen continuous improvements over traditional techniques. As the core of tissue repair, seed cells are widely used in various fields of regenerative medicine. However, their use is still associated with problems such as decreased cell survival and regeneration capacity after transplantation, immune rejection, and ethical concerns. Therefore, it is difficult to universally and safely apply stem cell banks for regenerative medicine. The paracrine effects of cells, especially secretion of exosomes, play vital roles in cell communication, immune response, angiogenesis, scar formation, tissue repair, and other biological functions. Exosomes are a type of nanoscale extracellular vesicle that contain biologically active molecules such as RNA and proteins; therefore, exosomes can replicate the functions of their parental cells. Meanwhile, exosomes can be used as nanocarriers to deliver active factors or small molecules to promote tissue repair. Preclinical studies of exosomes in tissue engineering and regenerative medicine have been carried in the fields of bone/cartilage repair, nerve regeneration, liver and kidney regeneration, skin repair, vascular tissue regeneration, etc. This review introduces exosomes from the aspects of biogenesis, composition, identification, and isolation, and focuses on the development status of scaffold materials for exosome delivery. In addition, we highlight examples of exosome-laden scaffolds for preclinical applications in tissue repair. We look forward to the broad application prospects of exosomeladen scaffolds.
\end{abstract}

Received 28th February 2021 Accepted 26th April 2021

DOI: $10.1039 / \mathrm{d} 1 \mathrm{nr} 01314 \mathrm{a}$

rsc.li/nanoscale received increasing attention. Recent studies have found that mesenchymal stem cells (MSCs) secrete exosomes (MSC-exos) containing biological molecules that play vital roles in tissue repair. As a cell-free biomaterial, exosomes can partially solve the problems encountered in clinical applications of regenerative medicine, such as the source, quantity, and immune rejection of seed cells. Therefore, combining exosomes with tissue engineering scaffold materials can provide a new generation of scaffold biomaterials that are better suited for tissue repair. In this review, we summarize the functions of exosomes in tissue repair, the state-of-the-art materials used for exosome tissue engineering, and preclinical applications. Moreover, we provide a perspective on the prospects of exosome-based tissue engineering for tissue repair.

\section{Biogenesis and composition of exosomes}

Cells secrete a variety of extracellular vesicles (EVs), which can be divided into microvesicles, apoptotic bodies, exosomes, etc. according to their biogenesis, size, density, and major protein markers. Exosomes are a subtype of saucer-shaped vesicles 40-160 $\mathrm{nm}$ in diameter that float at a density of $1.13-1.19 \mathrm{~g}$ 
$\mathrm{ml}^{-1}$ in sucrose gradients. Exosomes are secreted and taken up by various cell types such as endothelial cells, immune cells, tumor cells, and MSCs. Therefore, exosomes are a main intermediary in cell-to-cell communication.

Unlike microvesicles, which are formed by outward budding and fission of the plasma membrane, exosome biogenesis begins with inward budding of the plasma membrane to form primary endocytic vesicles (Fig. 1). Multiple primary endocytic vesicles fuse to form early endosomes (EEs) through clathrin- or small porin-dependent or -independent pathways to form multiple intraluminal vesicles (ILVs) encapsulating cytosolic components. ILVs further form late endosomes (LEs), also called intracellular multivesicular bodies (MVBs). Then, with the assistance of soluble $N$-ethylmaleimide-sensitive factor attachment protein (SNARE), Rab27A and Rab27B regulate fusion of MVBs with the plasma membrane to promote release of exosomes into the extracellular space by exocytosis. $^{1,2}$ At present, it is believed that the production of exosomes involves two pathways: the endosomal sorting complex required for transport (ESCRT)-dependent pathway and the ESCRT-independent pathway. The ESCRT-dependent pathway is widely accepted as a key process in exosome biogenesis and cargo sorting. ESCRT consists of four complexes: ESCRT-0 (HRS), ESCRT-I (TSG101, VPS28, VPS37), ESCRT-II (VPS22, VPS36, VPS25), and ESCRT-III (ALIX, VPS2). ${ }^{3}$ ESCRT-0, ESCRT-I, and ESCRT-II complexes recognize ubiquitinated proteins in the endosomal membrane, while ESCRT-III complex regulates membrane budding and vesicle separation. ${ }^{1,4}$ ESCRT guides specific molecules into MVBs to ensure exosome secretion. ${ }^{5,6}$ Studies have found that depletion of ESCRT not only changes the protein composition of exosomes but also reduces exosome secretion. However, ESCRT depletion does not completely block exosome biogenesis, indicating that an ESCRT-independent pathway, such as lipid raft-mediated pathway or ceramide-dependent pathway, also plays a role in exosome biogenesis and cargo sorting. ${ }^{7,8}$ More interest, the structure and environmental impact of biological materials can also affect the functional types and functions of exosomes

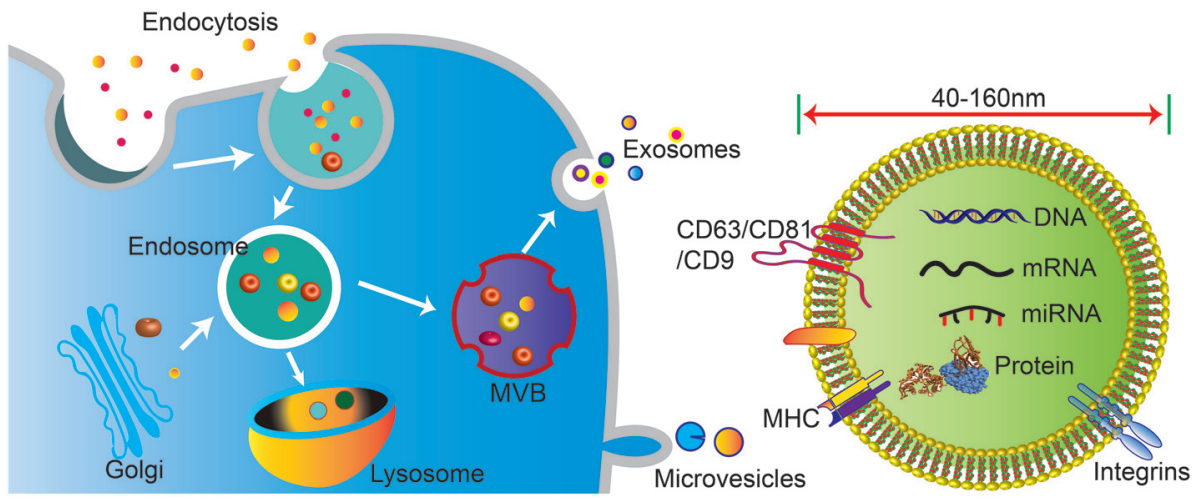

Fig. 1 Schematic illustration of exosome biogenesis and composition. Exosome formation is initiated by invagination of the plasma membrane to form EEs, which fuse to form MVBs. Then, MVBs fuse with the plasma membrane to release exosomes into the extracellular matrix, or fuse with lysosomes for degradation. In comparison, microvesicles are formed directly by outward budding of the plasma membrane. The composition of exosomes includes lipids, DNA, RNA, cytosolic proteins, and surface membrane proteins.

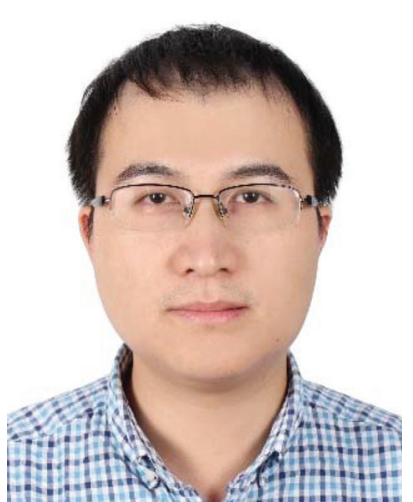

Jianghong Huang
Jianghong Huang is carrying out his Ph.D. of innovation leading engineering at Tsinghua University Shenzhen International Graduate School, supervised by Prof. Shuqing Sun. While he worked as a research scientist at the department of orthopedics, Shenzhen Second People's Hospital. His research interests focus on design and synthesis of biocompatible $3 D$ scaffolds for tissue engineering and regenerative medicine.

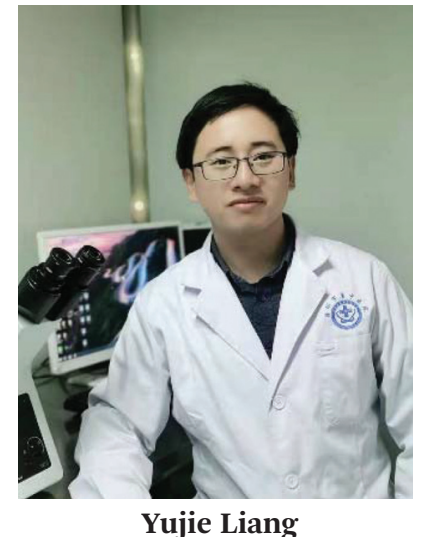

Yujie Liang
Yujie Liang obtained his Ph.D. in Chemistry at The Chinese University of Hong Kong. Previously, he was a research fellow at Peking University and Peking University Shenzhen Graduate School. His current work is as principal investigator at the Department of Child and Adolescent Psychiatry, Shenzhen Kangning Hospital, Shenzhen Mental Health Center. His research focuses on engineering exosomes for targeted therapy. 
released by cells. For example, a hydrogel composed of PG and TCP impacted the microenvironment of rat bone marrowderived MSCs, leading to secretion of exosomes to induce angiogenesis. ${ }^{9,10}$

Exosomes contain a variety of biologically active substances from their parental cells, which reflects their functions in recipient cells. According to the results of multi-omics research, the composition of exosomes includes three major types of biomolecules: nucleic acids, proteins, and lipids. Specifically, MSC-derived exosomes not only express common surface markers, but also express MSC characteristics like CD29, CD44, CD73, CD90, and CD105. Exosomes derived from bone marrow mesenchymal stem cells (BMSC) were shown to express 730 functional proteins, including those related to tissue regeneration, such as angiogenesis, blood coagulation, apoptosis, inflammation, and extracellular matrix remodeling, although they lack nucleoprotein. MSCs exosomes also contain cytokines such as VEGF, TGF- $\beta 1$, IL-6, IL-10, and HGF, which are beneficial to angiogenesis and immune regulation.

Exosomes are enriched in a variety of miRNAs, which regulate the function and activity of target cells and organs. The miRNA encapsulated into exosomes can regulate gene expression, and its ratio is higher than that in cells. For example, miR-155, let-7f, miR-199a, miR-221, miR-125b-5p, miR-22, among others, participate in a variety of physiological and pathological processes. Although exosomes inherit biological components from parental cells, the miRNA content in exosomes is higher than that of parental cells, indicating that entry of miRNAs into exosomes is regulated. Gibbings et al. found that exosomes contain GW182 and AGO2, which promote the continuous assembly or disassembly of membrane-associated miRISC that is necessary for miRNA loading or target recognition and subsequent silencing. ${ }^{11}$ Another study demonstrated that neutral sphingomyelinase-dependent pathway is related to miRNA load in exosomes. ${ }^{12}$ However, the study of functional miRNA in exosomes faces several challenges. First, the sorting mechanism by miRNA enter exosomes is unclear. Second, it is unknown whether functional miRNAs and proteins act independently or synergistically, therefore highlighting the need for further research.

Other non-coding RNAs, including IncRNA, circRNA, ribosomal RNA, transfer RNA, small nucleolar RNA, small nuclear RNA, and piwi-interacting RNA, are secreted by the parental cell and transported to recipient cells via exosomes, where they can perform special functions. In addition, nucleic acid components of exosomes, such as DNA and mRNA, can be translated into proteins after entering the cytoplasm of recipient cells to initiate their function.

Exosomes are different from other lipid nanoparticles in that their surface is rich in membrane proteins, which can mediate adhesion and targeting functions between exosomes and the plasma membrane of recipient cells, thereby regulating exosome uptake. ${ }^{13}$ Tetraspanins (CD9, CD63, and CD81), endosomal origin proteins (TSG101 and ALIX), MVB formation-related proteins (flotillin and annexin), and heat shock proteins (HSP70 and HSP90) are common biomarkers of exosomes. ${ }^{14,15}$ In addition, there are a variety of immune cellrelated proteins on the exosomal membrane such as major histocompatibility complex (MHC) class I and II proteins, which are involved in the processing and presentation of antigens. ${ }^{16}$ Exosomes derived from different cell sources or under different physiological and pathological conditions will encapsulate a variety of cell type-specific proteins, indicating that the recruitment or inclusion of exosomal cargo may be a dynamic regulatory process. This can form the basis on which specific markers for liquid biopsy are developed. In addition, exosomes are rich in cholesterol, sphingomyelin, glycosphingolipid, phosphatidylserine, and ceramide. The lipid content is conservative and essential for maintaining the exosomal morphology, generating exosomes, and regulating homeostasis. In short, exosomes contain specific substances from the parental cell that can be used as interventional targets for tissue repair and regeneration, immune regulation, cancer treatment, clinical disease diagnosis, and other applications. Moreover, the engineering of exosomes can result in improved function, which can be achieved through targeted modification or genetic modification of parental cells to overexpression and enrich the specific content of exosomes.

\section{Exosomes in tissue repair}

Exosomes can be absorbed by acceptor cells through autocrine or paracrine pathways and are able to migrate long distances to target tissues or organs through the circulatory system to participate in various physiological and pathological processes of tissue repair. Exosomes can deliver their cargo into the cytosol of recipient cells through non-specific pathways, such as macropinocytosis or micropinocytosis, or by specific receptor-mediated processes. ${ }^{17,18}$ Exosomes can also activate intracellular signaling pathways to trigger repair of target cells by directly acting at the cell surface without transferring their cargo. ${ }^{19}$ Exosomes are also natural nanocarriers. Their phospholipid bilayer structure protects biologically active substances, such as growth factors and miRNA, for sustained release. Due to their nanosize, exosomes can escape phagocytes and freely shuttle between cells or matrixes. They also have a strong penetrating ability and low immunogenicity. Exosomes can also be loaded with biological macromolecules, short peptides, miRNAs, and small molecule drugs simultaneously to enhance their tissue repair capabilities.

MSCs are the main source of exosomes (MSC-exos) for tissue repair. Many groups have demonstrated that MSC-exos protect myocardium during ischemia-reperfusion injury, ${ }^{20-22}$ relieve limb ischemia, ${ }^{23}$ promote wound healing, ${ }^{24}$ alleviate kidney damage, ${ }^{25}$ stimulate liver regeneration, ${ }^{26}$ promote neuronal regeneration, ${ }^{27}$ and enhance cartilage tissue ${ }^{28-31}$ and bone tissue regeneration ${ }^{32,33}$ (Fig. 2). MSC-exos contain markers commonly expressed by all exosomes (including CD9, CD81, CD63, TSG101, ALIX, tubulin, and actin) as well as MSC surface markers (CD29/CD90/CD73). MSC-exos contain unique miRNA including miR-191/miR-222/miR-21/let-7a (regulate cell 


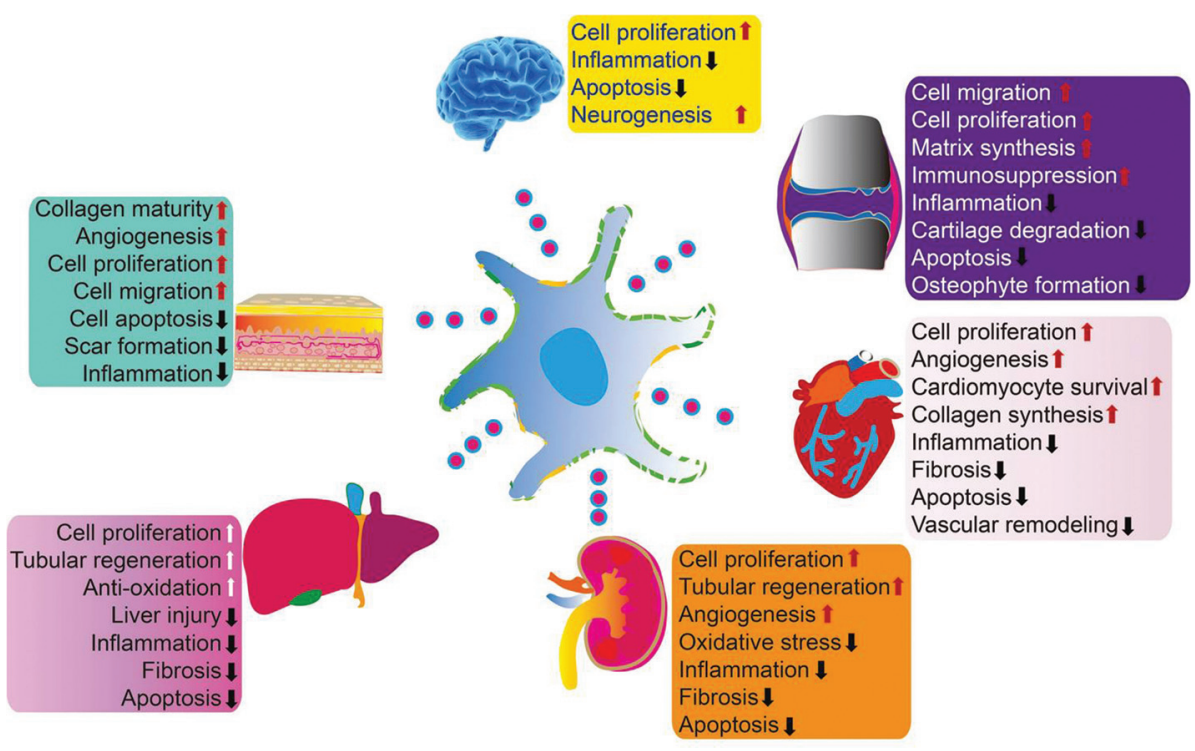

Fig. 2 MSC-exos have demonstrated beneficial effects in multiple organs and tissues by enhancing cell proliferation, attenuating inflammation, apoptosis, and macrophage responses, modulating oxidative stress, and other mechanisms. Reprinted with permission from ref. 39 , copyright 2016 CC-BY.

proliferation), ${ }^{34}$ miR-222/miR-21/let-7a (promote angiogenesis), ${ }^{35}$ miR-6087 (promotes endothelial differentiation), ${ }^{36}$ miR-494 (promotes muscle growth), ${ }^{37}$ miR-10b (promotes cell migration), ${ }^{38}$ and $\mathrm{miR}-181 \mathrm{c} / \mathrm{miR}-146 \mathrm{a} / \mathrm{miR}-548 \mathrm{e}$ (reduce inflammation). ${ }^{28,39,40}$

\section{Advantages and limitation of exosomes for tissue repair}

The emergence of MSC-based tissue engineering approaches for clinical therapeutics has been an exciting and new innovation. However, concerns about their instability and potential to form cancers have been revealed. ${ }^{41}$ These concerns have made the research community reconsider the biological safety of stem cell therapies. With the advent of cell-free therapies, exosomes have emerged as tools for tissue regeneration that may overcome the limitations and risks of traditional stem cell therapies. Studies have shown that MSC-exos have similar functions as MSCs, including repairing and regenerating tissues, inhibiting inflammation, and regulating immunity. The use of MSC-exos for tissue repair has several potential advantages. First, they can avoid the risk of immunity caused by stem cell transplantation. Second, exosomes can be stored for a long time and can be utilized at any time, which provides convenience for rapid clinical application. Third, the vesicles are small and so can circulate through capillaries, unlike MSCs. In particular, exosomes can enter the lungs to promote lung repair after COVID-19. ${ }^{42}$ However, exosomes are rapidly cleared from the blood, after entering the blood circulation, exosomes are quickly eliminated from blood vessels and enter the parenchymal organs. ${ }^{43}$ The plasma half-life of exosomes was found to be only 2-4 $\min .{ }^{44}$ Further research found that the accumulation of exosomes in the liver, spleen, lung, and gastrointestinal tract can be found as early as $2 \mathrm{~h}$ after systemic injection, ${ }^{45,46}$ this fate of exosomes was primarily phagocytosis by macrophages in the liver and spleen. Inhibiting the activity of macrophages can significantly prolong the plasma half-life of exosomes. ${ }^{47}$ This process may be due to the expression of phosphatidylserine on exosomes, an apoptotic signal which results in the recognition and subsequent phagocytosis by macrophages ${ }^{48}$ Additionally, exosomes are rapidly cleared from tissues. Therefore, implantable biomaterial scaffolds have been developed for sustained therapeutic delivery of exosomes. For example, encapsulation of exosomes in hydrogel patches can prevent their premature tissue elimination and also promote local and concentrated release at or near the site of injury $^{49}$ (Fig. 2).

\section{Exosome-laden scaffolds}

The development of smart biomaterial scaffolds for efficient and continuous release of adsorbed exosomes is becoming a hot research area in tissue engineering. To efficiently promote wound healing, the ideal scaffold material for MSC-exos has the following characteristics: (1) effectively retains MSC-exos at the tissue defect site and maintains their activity and structural integrity; (2) releases MSC-exos into the matrix for a long time to regulate phenotype changes of the surrounding cells; (3) seamlessly integrates with the damaged tissue to promote migration of the surrounding cells into the scaffold as well as exosome homing. Once the cells have migrated into the scaffold, the MSC-exos can be absorbed by the cells and actively regulate and promote tissue repair and regeneration. 
At present, physical embedding and diffusion are the most commonly used methods for exosome loading. In particular, the dispersion of exosomes largely depends on the pore size and crosslinking density of the hydrogel. As the hydrogel has a porous microstructure, adjustable biophysical parameters and degradation rate, the swelling or degradation of the hydrogel matrix will increase the size of the polymer mesh, resulting in the sustained release of exosomes. Therefore, stronger interactions (such as covalent bonds and electrostatic interactions) between the matrix and exosomes can provide more effective protection and immobilization of exosomes. In this section, we summarize common methods for constructing exosomeladen scaffolds (Fig. 3).

\subsection{Methods for isolating exosomes}

The first step in constructing exosome-laden scaffolds is to obtain high-quality exosomes. At present, exosomes are isolated based on their size, density, and immunoaffinity by the following methods: ultracentrifugation, density gradient centrifugation, size-exclusion chromatography, polymer-based precipitation, microfluidic separation, and immunoaffinity capture. Ultracentrifugation is currently considered the gold standard, and most researchers utilize this exosome separation technique. However, ultracentrifugation has several shortcomings, including the need for a large volume of biological fluid, long processing times, and limited reproducibility. ${ }^{50}$ Compared with conventional isolation methods, microfiltration centrifugation can quickly, portably, and accurately process nanoparticles from small volumes of liquid samples. ${ }^{50}$
The advantages and disadvantages of commonly used isolation techniques for MSC-exos are listed in Table 1.

\subsection{Ionically crosslinked hydrogel scaffolds}

Several attempts have been made to design ionically crosslinked scaffolds for exosome retention and release. The representative material for these scaffolds is alginate (Alg), which is a substance extracted from seaweed such as brown algae, sea lichen, Japanese kelp, ascomycetes, and megasporidia. Alg is a linear copolymer containing (1,4)-D-mannuronic acid and L-guluronic acid residues. Alg hydrogel crosslinked with divalent cations (such as $\mathrm{Ca}^{2+}$ ) has recently been used as a cell delivery scaffold for tissue engineering applications. ${ }^{51}$ The structure of the guluronic acid block allows a wide range of divalent cations to coordinate with the Alg chain. This forms ionic bridges between guluronic acid blocks of adjacent polymer chains to form an egg-box-like structure. Alg hydrogel is a relatively common type of scaffold for MSC-exos. For example, Shafei et al. prepared an Alg exosome-laden scaffold for skin wound repair. ${ }^{52}$ Compared with Alg scaffold alone, the exosomes-loaded scaffold greatly enhanced skin wound closure, collagen synthesis, and angiogenesis in the wound area. Therefore, it was proposed as an ideal bioactive composite dressing for the treatment of skin injuries. In another study, an Alg scaffold loaded with exosomes isolated from human umbilical cord-derived MSCs was developed to treat pain caused by nerve injury. ${ }^{53}$ Due to its high biocompatibility, biodegradability, non-antigenicity, and high-water absorption properties, Alg is considered to be a suitable functional material for biomedical applications.
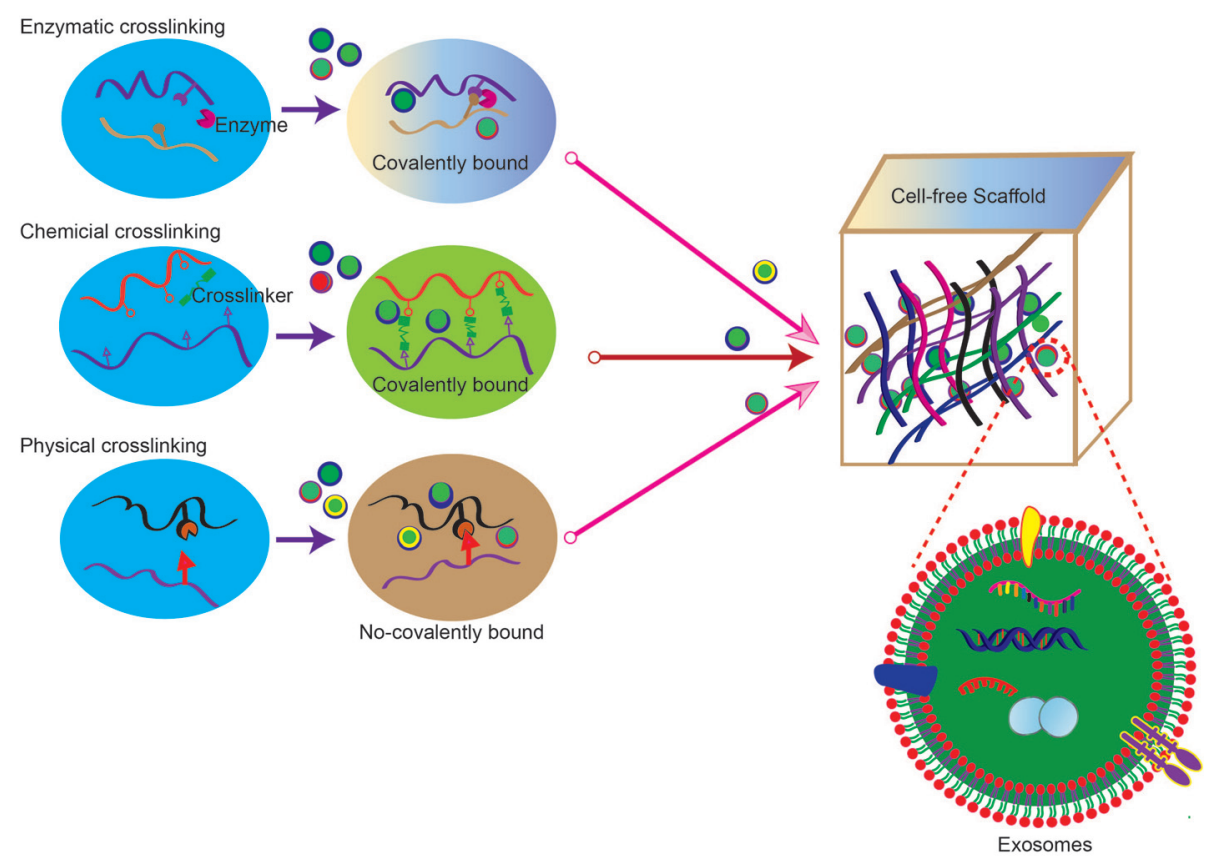

Fig. 3 Strategies commonly utilized for constructing exosome scaffolds. Hydrogel synthesis by polymer-polymer interactions with chemical crosslinking, enzymatic crosslinking, and physical crosslinking methods are illustrated. Exosomes are incorporated into the hydrogel before, during, or after crosslinking. 
Table 1 Exosome isolation methods

\begin{tabular}{|c|c|c|c|}
\hline Method & Principle & Advantage & Disadvantage \\
\hline Ultracentrifugation & Density, size, and shape & Low cost, high quality & $\begin{array}{l}\text { Large sample volume, expensive } \\
\text { equipment, time consuming }\end{array}$ \\
\hline Chromatography & $\begin{array}{l}\text { Molecular size or molecular } \\
\text { weight }\end{array}$ & Fast, high purity, direct extraction of RNA & $\begin{array}{l}\text { Large influence of external forces, } \\
\text { low yield }\end{array}$ \\
\hline Microfluidics & $\begin{array}{l}\text { Physical and biochemical } \\
\text { properties }\end{array}$ & Fast, low cost, automated & $\begin{array}{l}\text { No standardization, no clinical } \\
\text { testing }\end{array}$ \\
\hline Immunoaffinity & $\begin{array}{l}\text { Exosomal antigen and antibody } \\
\text { interaction }\end{array}$ & High purity & High cost, time consuming \\
\hline Precipitation & Solubility and isoelectric point & Convenient, no special equipment required & Low purity \\
\hline $\begin{array}{l}\text { Microfiltration } \\
\text { centrifugation }\end{array}$ & $\begin{array}{l}\text { Combination of ultrafiltration } \\
\text { with ultracentrifugation }\end{array}$ & $\begin{array}{l}\text { State-of-art equipment, high output, low } \\
\text { consumption, relatively integrity of } \\
\text { exosomes }\end{array}$ & $\begin{array}{l}\text { Exosomes may adhere to the } \\
\text { filtration membranes }\end{array}$ \\
\hline
\end{tabular}

\subsection{Photo-crosslinked scaffolds}

Photopolymerization is a type of free radical polymerization induced by a photoinitiator under ultraviolet or visible light irradiation. Commonly used materials for the preparation of photo-crosslinked hydrogels are chitosan and gelatin. Chitosan is the second most abundant natural polysaccharide. It is a polycationic polymer that consists of repeating units of $N$-acetyl-D-glucosamine and D-glucosamine. Chitosan is highly biocompatible, nontoxic, biodegradable, nonimmunogenic, and mucoadhesive. Gelatin is one of the main components of cartilage. It is a protein product mainly derived from collagen by denaturation and hydrolysis. Gelatin has good cell adhesion properties and biodegradability. Therefore, scaffolds containing gelatin matrix have natural advantages in promoting cell migration, proliferation, and differentiation and inducing cellmediated enzymatic degradation. ${ }^{54}$ Because of their advantageous properties, chitosan and gelatin materials have been extensively used in many fields. Since gelatin is unstable when exposed to heat, it is necessary to synthesize stable biomaterials by covalent crosslinking. As chitosan cannot be directly polymerized to form a hydrogel, photochemical groups are introduced. For example, hybrid hydrogel scaffolds have been synthesized by introducing polymerizable groups (e.g., azidobenzoic acid, methacrylic acid, polyethylene glycol, carboxymethyl, acetocarboxyl) and a photoinitiator (commonly $\alpha$-hydroxyalkylphenone) to crosslink chitosan with gelatin under ultraviolet light. ${ }^{55}$ Another study achieved visible lightinduced polymerization using methacrylated glycol chitosan and blue light initiators such as riboflavin, camphorquinone, and fluorescein. ${ }^{56}$ The authors developed an injectable photocrosslinked hydrogel to deliver human MSC-derived EVs for bone repair. In a mouse skull defect model, the scaffold showed excellent bone repair performance. Similarly, gelatin methacryloyl hydrogels with controllable photo-crosslinking have been reported.

\subsection{D bioprinted scaffolds}

3D printing is an additive manufacturing technology that allows selective distribution of cells, biological materials, and growth factors to produce 3D living tissues and organs. These scaffolds are characterized by their hierarchical structures or intelligent surfaces, which can control exosome activity and structure. In one study, researchers modified 3D-printed titanium alloy scaffolds with exosomes that induce osteogenic differentiation of human MSCs for cell-free bone regeneration to avoid the immune rejection and teratoma formation observed with stem cells. ${ }^{57}$ Chen et al. generated a cartilage extracellular matrix/gelatin methacrylate exosome-laden scaffold through a light-curing 3D printing process. ${ }^{58}$ The endosome scaffold effectively restored chondrocyte mitochondrial dysfunction, strengthened cartilage, and significantly promoted cartilage regeneration in rabbit articular cartilage defects. Kim et al. prepared 3D porous silk fibroin scaffolds and demonstrated that exosomes isolated from human adipose-derived MSCs can induce osteogenic differentiation and bone regeneration in vitro and in vivo. ${ }^{59}$ Recent research has shown that modified exosomes combined with a $3 \mathrm{D}$ printed porous bone scaffold through a specific linker (exosomal anchor peptide CP05) can effectively increase the osteogenesis and angiogenesis by exosomes encapsulating the VEGF gene. ${ }^{60}$

\subsection{Tissue engineering applications of exosome-laden scaffolds}

The short tissue retention of exosomes after in vivo implantation is still a major challenge in clinical applications. Hydrogel encapsulation of exosomes can enable continuous delivery in the injured environment, thereby improving the therapeutic effect. Table 2 summarizes the most commonly used hydrogels for exosome delivery and their applications.

Researchers have developed a number of methods that can sustainably deliver exosomes to the post-infarct environment. For example, exosomes isolated from cardiomyocyte-derived induced pluripotent stem cells encapsulated in hydrogel patches were directly delivered to the hearts of infarcted rats. ${ }^{61}$ The exosome patches demonstrated prolonged exosome release and promoted recovery of the ejection fraction, prevented cardiomyocyte hypertrophy, alleviated the ischemic injury, and promoted recovery of the heart. Another study loaded endothelial progenitor cell-derived exosomes into a shear-thinning gel to achieve precise administration and sustained delivery. ${ }^{62}$ In a rat model of myocardial infarction, the 
Table 2 Examples of exosome-laden scaffolds and their applications in tissue repair

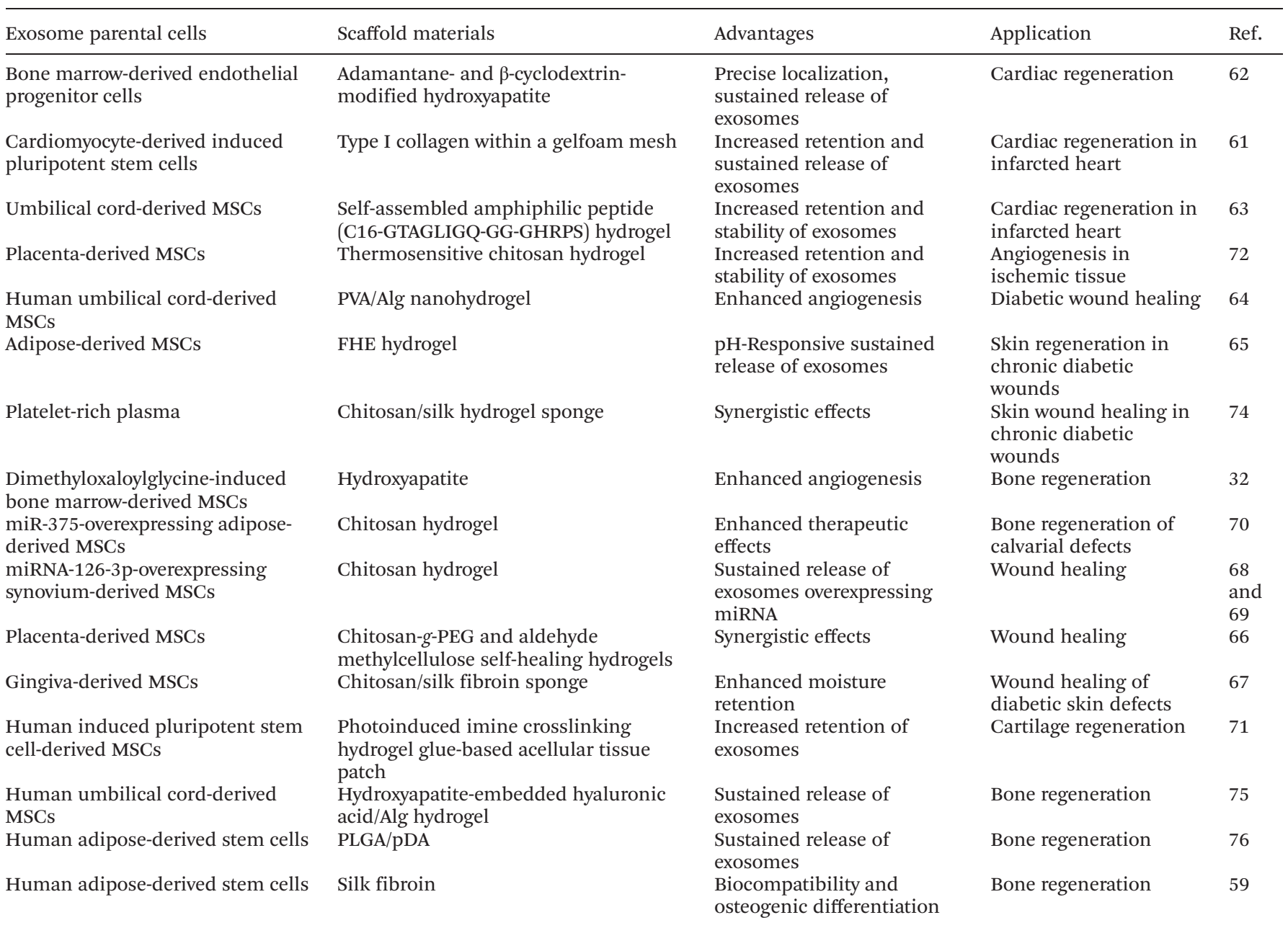

exosome hydrogels enhanced angiogenesis and myocardial hemodynamics around the infarct. The cell-free scaffold material improved the effects of exosome-mediated myocardial therapy. In another study, exosomes isolated from human umbilical cord-derived MSCs were encapsulated in functional peptide hydrogels to increase their stability and provide sustained release. ${ }^{63}$ The exosome hydrogels protected cardiomyocytes from oxidative stress induced by $\mathrm{H}_{2} \mathrm{O}_{2}$, which improved cardiac function in a rat myocardial infarction model. These studies provide practical and effective methods for the use of exosome-laden scaffolds in myocardial regeneration.

Exosome-laden scaffolds are most widely used for skin repair. Several findings indicate that the combination of bioactive scaffold materials with controlled release of exosomes heals skin wounds. For example, exosomes isolated from human umbilical cord-derived MSCs encapsulated in polyvinyl alcohol (PVA)/Alg nanohydrogels were used to heal diabetic wounds. ${ }^{64}$ The PVA/Alg nanohydrogel promoted cell proliferation, migration and angiogenesis, enhanced the efficacy of exosomes, and accelerated healing of diabetic wounds. In another study, exosomes were loaded in a novel injectable bioactive hydrogel called FHE. ${ }^{65}$ This hydrogel was composed of oxidative hyaluronic acid, which provided water retention and biocompatibility, poly- $\varepsilon$-L-lysine, which provided antibacterial activity and adhesion, and Pluronic F127, which provided thermal responsiveness. FHE hydrogel exhibited inherent antibacterial activity, self-repair, and $\mathrm{pH}$-sensitive release of exosomes. Exosomes isolated from adipose-derived MSCs were loaded into FHE hydrogel through electrostatic interactions with poly- $\varepsilon$-L-lysine. The exosome hydrogel promoted angiogenesis, cell proliferation, and granulation tissue formation at the wound site and accelerated the healing of diabetic wounds and skin regeneration. In another study, methylcellulose-chitosan hydrogels loaded with exosomes isolated from placentaderived MSCs were shown to heal diabetic wounds and achieved formation of new tissues similar to natural skin. ${ }^{66}$ Similarly, chitosan/silk hydrogels with swelling and moisturizing capabilities loaded with exosomes isolated from gingivaderived MSCs promoted collagen epithelial regeneration and angiogenesis and accelerated the healing of diabetic skin defects. ${ }^{67}$ Chitosan scaffolds have also been shown to provide controlled release of exosomes isolated from synovium-derived MSCs, which accelerated wound healing by increasing the formation of granulation tissue and angiogenesis. ${ }^{68,69}$ Using gene 
overexpression technology, the angiogenesis promotion ability of endothelial progenitor cells was transferred to the exosomes via miR-126-3p. This study shows that exosomes can be loaded with nucleic acid drugs to increase their potential for tissue repair, which may provide optimization space for future treatments. In summary, highly efficient, self-repairing, and biocompatible natural hydrogels loaded with MSC-exos have been prepared by simple methods for the treatment of severe diabetic skin wounds.

Modified exosomes also have the potential to stimulate bone regeneration. For example, miR-375 was enriched in exosomes by overexpression in parental cells. ${ }^{70}$ The exosomes were loaded into a hydrogel, which was injected into a rat skull defect model. The exosomes were continuously released into the wound, which enhanced bone regeneration. In another study, exosomes isolated from human bone marrow-derived MSCs stimulated by dimethyloxaloylglycine were loaded into a porous hydroxyapatite scaffold to improve bone proangiogenic activity in bone healing. ${ }^{32}$ Liu et al. developed a photoinduced imine crosslinking hydrogel glue to generate a decellularized tissue patch for cartilage regeneration. ${ }^{71}$ The patch retained stem cell-derived exosomes in the cartilage for a long time. In addition, the exosome-laden scaffold integrated with the natural cartilage matrix, induced cell migration in the cartilage defect, and promoted the repair and regeneration of articular cartilage. Another study constructed a cell-free bone tissue engineering system by combining poly(lactic-co-glycolic acid) (PLGA)/polydopamine (pDA) scaffolds and exosomes isolated from human adiposederived stem cells. ${ }^{61}$ The exosomes were slowly and continuously released from the scaffold, which promoted migration of MSCs and significantly enhanced bone regeneration.

Exosome-laden scaffolds have also been developed for other applications. For example, chitosan hydrogel scaffolds were developed to enhance the therapeutic effects of exosomes on hindlimb ischemia and improve tissue regeneration after ischemic injury. ${ }^{72}$ Xin et al. loaded collagen scaffolds with exosomes for endometrial regeneration in a rat endometrial injury model. ${ }^{73}$ This bioactive scaffold was biodegradable and biocompatible and significantly improved exosomal functions.

\section{Future directions and conclusions}

A large number of exosome-laden scaffolds have been investigated preclinically for the repair and regeneration of bone, cartilage, skin, heart, liver, and kidney tissues, demonstrating the therapeutic potential of exosomes. An important aspect of exosomes as cell-free therapies is immune regulation. According to many studies, exosomes have a strong effect on humoral immunity and cellular immunity, and may have anti-inflammatory and pro-inflammatory functions related to tissue repair and regeneration after injury. Another aspect that cannot be ignored is the role of exosomes in signal transmission between cells. Exosomes can change the movement, proliferation, phenotype, and maturation of cells. They can also maintain cells by spreading protective or damage signals.
Exosomes can used use as drug delivery vehicle. Although their size and a phospholipid structure are similar to those of liposomes, natural derived exosomes have multiple advantages over other nanoparticles. Specifically, naturally-formed exosomes exhibit favorable biocompatibility, biodegradability, as well as low toxicity and immunogenicity. Additionally, other studies have shown that the exosomes themselves have cellular selectivity and tissue specificity, as well as the ability to cross the blood-brain barrier and penetrate its dense structure. Similarly, methods for the surface modification of liposomes can also be applied to the functionalized modification of exosomes. All in all, this naturally-derived exosome holds great potential in drug delivery. However, there are still many hurdles for exosomes. For example, although ultracentrifugation is the preferred method for exosome isolation, it still has disadvantages such as low purity and easy degradation of exosomes. Although some commercial reagents can increase the exosome isolation yield, the purity is reduced and new isolation methods still need to be developed. The scale-up method for the purification of exosomes is challenging. Preparation of high-purity and high-yield exosomes is a key bottleneck to their clinical application in tissue repair. At present, the main methods for isolation of GMP-level exosomes are differential ultracentrifugation (UC) and microfiltration platforms. This is because the higher shearing forces of UC will destroy exosomes and induce EV aggregation, which then leads to the release of proteins from the exosomes. In recent years, tangential flow filtration (TFF) has arisen as the most promising solution for the concentration and purification of exosomes. This is due to the ability of TFF to purify the required exosomes on a large scale. In addition, the exosomes collected from TFF contain more soluble cytokine and shown higher immunomodulatory ability than those from the ultracentrifugation method. ${ }^{77}$ Therefore, tangential flow filtration (TFF) will be suitable for mass production of highquality exosomes in compliance with GMP for tissue repair. Recently, the higher yield of exosome-mimetic nanovesicles with very similar properties to naturally-formed exosomes could extend the prospects for application. ${ }^{78}$ Although there has been much progress in the targeted modification of exosomes, the in vivo environment can introduce complexities to the disposition of the modified exosomes. Thus, whether the modified exomes can cause an immune response should also be further investigated. In addition, the mechanisms by which exosomes repair and regenerate tissues have not been fully elucidated. It is not clear which components/properties of exosomes promote tissue regeneration. The therapeutic exosome dose also needs to be optimized, as excessive exosomes may cause irreversible tissue damage.

Exosome-laden scaffolds provide a feasible solution for achieving continuous release of exosomes in tissue repair. Because hydrogel has the characteristics of high tissue-like water content, easy implantation, and high biocompatibility, it is increasingly used as a carrier of exosomes for tissue engineering. The optimization of materials, such as the use of HGM gelatin supramolecular hydrogels, can promote the sustainable 
(A)

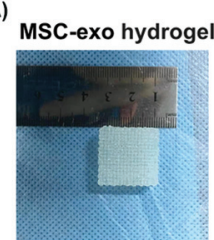

(C) MSC-exo/Dilc

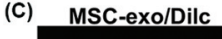

release of loaded small molecules and proteins, thereby enhancing the cartilage formation effect of MSC. $^{79}$ Furthermore, HA-pamidronate-grafted HA (HA-Pam) hydrogel can mediate the sustained release of Dex through positive feedback triggered by ALP, further promoting the osteogenic differentiation of encapsulated hMSC. ${ }^{80}$ These new hydrogels can also be utilized as exosomal scaffold materials, and injectable hydrogels are especially ideal for use as exosomal scaffold carriers in in vivo tissue repair. ${ }^{81}$ As shown in Fig. 4, we former developed a gelatin/hydroxyapatite scaffolds that can also use for exosome coat. ${ }^{82}$ This result shown continuously release encapsulated exosomes into chondrocytes over 6 days, indicated that the hydrogel effectively retained the exosomes. Hydrogels such as Alg, PLGA, pDA, and FHE can be applied in hard and soft tissue regeneration. Strategies for encapsulating stem cell-derived exosomes in hydrogels are still in the early stage. Challenges include the potential toxicity of residual unreacted crosslinking agents from hydrogel manufacturing, especially for injectable hydrogels designed to polymerize within tissues. When injecting $\mathrm{pH}$-sensitive or temperaturesensitive hydrogels, needle plugging may occur. Therefore, it is necessary to optimize the gelation temperature, polymer concentration, and applicator system to prevent premature gelation in the syringe. There are continuing challenges in deter- mining the kinetic release profile of exosomes from scaffolds in vivo. The release profile generated in vitro is usually not equivalent to the in vivo profile. Of course, exosomes can also be engineered with enhanced ability to target specific cells or tissues. For example, genetically modified exosomes were developed for targeted delivery of nucleic acids and small molecules to chondrocytes and MSCs, respectively, which greatly enhanced their ability to repair cartilage tissue. ${ }^{83-85}$ While dispersing exosomes into the scaffold material can significantly improve the local and sustained release of the exosomes. Loading the exosomes and cells at the same time yields more advantageous results than the traditional cell composite material. ${ }^{86}$ The drug loaded on the exosomes can be continuously released into the material, ensuring the maximum effect of the drug encapsulated in the exosomes. In conclusion, bioactive exosome-laden scaffolds are effective cell-free alternatives to cell-based tissue engineering.

\section{Author contributions}

J.H., J.Y., L.Y., J.Z and S.Q performed discussion; Y.L. drew the figures and conceptualized the manuscript; J.H. and Y.L. wrote the manuscript.

\section{Conflicts of interest}

The authors have declared no competing interests.

\section{Acknowledgements}

This work was supported by Shenzhen Science and Technology Projects (GJHZ20190820115203714, JSGG20191129094218565, JCYJ20200109150700942, JCYJ20180306170922163). Key Realm R\&D Program of Guangdong Province (2019B030335001), Summing Project of Medicine in Shenzhen (SZSM201612079). Shenzhen Fund for Guangdong Provincial High level Clinical Key Specialties (No. SZGSP013, SZGSP007). Shenzhen Key Medical Discipline Construction Fund (SZXK042 and SZXK049). Guangdong Basic and Applied Basic Research Foundation (No. 2020A1515011581, 2021A1515010985). We thanked Dr Danielle for checking spelling and grammatical errors of this manuscript.

\section{References}

1 A. Bobrie, M. Colombo, G. Raposo and C. Théry, Traffic, 2011, 12, 1659-1668.

2 N. P. Hessvik and A. Llorente, Cell. Mol. Life Sci., 2018, 75, 193-208.

3 I. Roxrud, H. Stenmark and L. Malerød, Biol. Cell, 2010, 102, 293-318.

4 J. H. Hurley and P. I. Hanson, Nat. Rev. Mol. Cell Biol., 2010, 11, 556-566. 
5 M. Colombo, C. Moita, G. van Niel, J. Kowal, J. Vigneron, P. Benaroch, N. Manel, L. F. Moita, C. Théry and G. Raposo, J. Cell Sci., 2013, 126, 5553-5565.

6 T. Wollert, C. Wunder, J. Lippincott-Schwartz and J. H. Hurley, Nature, 2009, 458, 172-177.

7 A. de Gassart, C. Geminard, B. Fevrier, G. Raposo and M. Vidal, Blood, 2003, 102, 4336-4344.

8 K. Trajkovic, C. Hsu, S. Chiantia, L. Rajendran, D. Wenzel, F. Wieland, P. Schwille, B. Brügger and M. Simons, Science, 2008, 319, 1244-1247.

9 B. Zhang, J. Huang, J. Liu, F. Lin, Z. Ding and J. Xu, Mater. Sci. Eng., C, 2021, 111782, DOI: 10.1016/j.msec.2020.111782.

$10 \mathrm{X} . \mathrm{Xu}, \mathrm{L} . \mathrm{Xu}, \mathrm{P}$. Zhang, K. Ouyang, Y. Xiao, J. Xiong, D. Wang, Y. Liang and L. Duan, Oxid. Med. Cell. Longevity, 2020, 2020, 8865499.

11 D. J. Gibbings, C. Ciaudo, M. Erhardt and O. Voinnet, Nat. Cell Biol., 2009, 11, 1143-1149.

12 N. Kosaka, H. Iguchi, K. Hagiwara, Y. Yoshioka, F. Takeshita and T. Ochiya, J. Biol. Chem., 2013, 288, 10849-10859.

13 G. van Niel, G. D’Angelo and G. Raposo, Nat. Rev. Mol. Cell Biol., 2018, 19, 213-228.

14 J. Kowal, G. Arras, M. Colombo, M. Jouve, J. P. Morath, B. Primdal-Bengtson, F. Dingli, D. Loew, M. Tkach and C. Théry, Proc. Natl. Acad. Sci. U. S. A., 2016, 113, E968E977.

15 C. Théry, K. W. Witwer, E. Aikawa, et al., J. Extracell. Vesicles, 2018, 7, 1535750.

16 S. I. Buschow, B. W. van Balkom, M. Aalberts, A. J. Heck, M. Wauben and W. Stoorvogel, Immunol. Cell Biol., 2010, 88, 851-856.

17 M. Mathieu, L. Martin-Jaular, G. Lavieu and C. Théry, Nat. Cell Biol., 2019, 21, 9-17.

18 S. Horibe, T. Tanahashi, S. Kawauchi, Y. Murakami and Y. Rikitake, BMC Cancer, 2018, 18, 47.

19 M. T. Roefs, J. P. G. Sluijter and P. Vader, Trends Cell Biol., 2020, 30, 990-1013.

20 J. Zhao, X. Li, J. Hu, F. Chen, S. Qiao, X. Sun, L. Gao, J. Xie and B. Xu, Cardiovasc. Res., 2019, 115, 1205-1216.

21 R. C. Lai, F. Arslan, M. M. Lee, N. S. Sze, A. Choo, T. S. Chen, M. Salto-Tellez, L. Timmers, C. N. Lee, R. M. El Oakley, G. Pasterkamp, D. P. de Kleijn and S. K. Lim, Stem Cell Res., 2010, 4, 214-222.

22 F. Arslan, R. C. Lai, M. B. Smeets, L. Akeroyd, A. Choo, E. N. Aguor, L. Timmers, H. V. van Rijen, P. A. Doevendans, G. Pasterkamp, S. K. Lim and D. P. de Kleijn, Stem Cell Res., 2013, 10, 301-312.

23 G. W. Hu, Q. Li, X. Niu, B. Hu, J. Liu, S. M. Zhou, S. C. Guo, H. L. Lang, C. Q. Zhang, Y. Wang and Z. F. Deng, Stem Cell Res. Ther., 2015, 6, 10.

24 J. Zhang, J. Guan, X. Niu, G. Hu, S. Guo, Q. Li, Z. Xie, C. Zhang and Y. Wang, J. Transl. Med., 2015, 13, 49.

25 S. Bruno, C. Grange, M. C. Deregibus, R. A. Calogero, S. Saviozzi, F. Collino, L. Morando, A. Busca, M. Falda, B. Bussolati, C. Tetta and G. Camussi, J. Am. Soc. Nephrol., 2009, 20, 1053-1067.
26 C. Y. Tan, R. C. Lai, W. Wong, Y. Y. Dan, S. K. Lim and H. K. Ho, Stem Cell Res. Ther., 2014, 5, 76.

27 Y. Zhang, M. Chopp, X. S. Liu, M. Katakowski, X. Wang, X. Tian, D. Wu and Z. G. Zhang, Mol. Neurobiol., 2017, 54, 2659-2673.

28 S. Zhang, W. C. Chu, R. C. Lai, S. K. Lim, J. H. Hui and W. S. Toh, Osteoarthritis Cartilage, 2016, 24, 2135-2140.

29 S. Zhang, K. Y. W. Teo, S. J. Chuah, R. C. Lai, S. K. Lim and W. S. Toh, Biomaterials, 2019, 200, 35-47.

30 L. Duan, X. Xu, L. Xu, H. Chen, X. Li, M. Alahdal, Y. Xiao, Y. Liang and J. Xia, Curr. Med. Chem., 2020, DOI: 10.2174/ 0929867327666201118161232.

31 Y. Liang, X. Xu, L. Xu, I. Prasadam, L. Duan, Y. Xiao and J. Xia, J. Drug Targeting, 2021, 1-16.

32 B. Liang, J. M. Liang, J. N. Ding, J. Xu, J. G. Xu and Y. M. Chai, Stem Cell Res. Ther., 2019, 10, 335.

33 J. Zhang, X. Liu, H. Li, C. Chen, B. Hu, X. Niu, Q. Li, B. Zhao, Z. Xie and Y. Wang, Stem Cell Res. Ther., 2016, 7, 136.

34 N. Nagpal and R. Kulshreshtha, Front. Genet., 2014, 5, 99.

35 C. Urbich, A. Kuehbacher and S. Dimmeler, Cardiovasc. Res., 2008, 79, 581-588.

36 J. K. Yoo, J. Kim, S. J. Choi, H. M. Noh, Y. D. Kwon, H. Yoo, H. S. Yi, H. M. Chung and J. K. Kim, Stem Cells Dev., 2012, 21, 2049-2057.

37 Y. Nakamura, S. Miyaki, H. Ishitobi, S. Matsuyama, T. Nakasa, N. Kamei, T. Akimoto, Y. Higashi and M. Ochi, FEBS Lett., 2015, 589, 1257-1265.

38 F. Zhang, S. Jing, T. Ren and J. Lin, Mol. Med. Rep., 2013, 8, 1084-1088.

39 S. B. Fang, H. Y. Zhang, C. Wang, B. X. He, X. Q. Liu, X. C. Meng, Y. Q. Peng, Z. B. Xu, X. L. Fan, Z. J. Wu, D. Chen, L. Zheng, S. G. Zheng and Q. L. Fu, J. Extracell. Vesicles, 2020, 9, 1723260.

40 C. Yang, W. Lim, J. Park, S. Park, S. You and G. Song, Mol. Hum. Reprod., 2019, 25, 755-771.

41 C. T. Carson, S. Aigner and F. H. Gage, Nat. Med., 2006, 12, 1237-1238.

42 P. W. Askenase, J. Extracell. Vesicles, 2020, 10, e12004.

43 R. M. Schiffelers, I. A. Bakker-Woudenberg and G. Storm, Biochim. Biophys. Acta, 2000, 1468, 253-261.

44 S. C. Saunderson, A. C. Dunn, P. R. Crocker and A. D. McLellan, Blood, 2014, 123, 208-216.

45 Y. Takahashi, M. Nishikawa, H. Shinotsuka, Y. Matsui, S. Ohara, T. Imai and Y. Takakura, J. Biotechnol., 2013, 165, 77-84.

46 B. György, M. E. Hung, X. O. Breakefield and J. N. Leonard, Annu. Rev. Pharmacol. Toxicol., 2015, 55, 439464.

47 T. Imai, Y. Takahashi, M. Nishikawa, K. Kato, M. Morishita, T. Yamashita, A. Matsumoto, C. Charoenviriyakul and Y. Takakura, J. Extracell. Vesicles, 2015, 4, 26238.

48 R. E. Cocco and D. S. Ucker, Mol. Biol. Cell, 2001, 12, 919-930. 49 A. Marote, F. G. Teixeira, B. Mendes-Pinheiro and A. J. Salgado, Front. Pharmacol., 2016, 7, 231. 
50 X. Wu, S. A. A. Showiheen, A. R. Sun, R. Crawford, Y. Xiao, X. Mao and I. Prasadam, Theranostics: Methods and Protocols, Springer, New York, 2019, pp. 81-91.

51 G. T. Grant, E. R. Morris and A. David, FEBS Lett., 1973, 32, 195-198.

52 S. Shafei, M. Khanmohammadi, R. Heidari, H. Ghanbari, V. Taghdiri Nooshabadi, S. Farzamfar, M. Akbariqomi, N. S. Sanikhani, M. Absalan and G. Tavoosidana, J. Biomed. Mater. Res., Part A, 2020, 108, 545-556.

53 J. M. Hsu, S. J. Shiue, K. D. Yang, H. S. Shiue, Y. W. Hung, P. Pannuru, R. Poongodi, H. Y. Lin and J. K. Cheng, J. Pain Res., 2020, 13, 3257-3268.

54 P. E. Van den Steen, B. Dubois, I. Nelissen, P. M. Rudd, R. A. Dwek and G. Opdenakker, Crit. Rev. Biochem. Mol. Biol., 2002, 37, 375-536.

55 I. C. Carvalho and H. S. Mansur, Mater. Sci. Eng., C, 2017, 78, 690-705.

56 J. Hu, Y. Hou, H. Park, B. Choi, S. Hou, A. Chung and M. Lee, Acta Biomater., 2012, 8, 1730-1738.

57 F. Wei, M. Li, R. Crawford, Y. Zhou and Y. Xiao, Acta Biomater., 2019, 86, 480-492.

58 P. Chen, L. Zheng, Y. Wang, M. Tao, Z. Xie, C. Xia, C. Gu, J. Chen, P. Qiu, S. Mei, L. Ning, Y. Shi, C. Fang, S. Fan and X. Lin, Theranostics, 2019, 9, 2439-2459.

59 D. K. Kim, S. Lee, M. Kim, Y. Jeong and S. Lee, Chem. Eng. J., 2021, 406, 127080.

60 Y. Zha, Y. Li, T. Lin, J. Chen, S. Zhang and J. Wang, Theranostics, 2021, 11, 397-409.

61 B. Liu, B. W. Lee, K. Nakanishi, A. Villasante, R. Williamson, J. Metz, J. Kim, M. Kanai, L. Bi, K. Brown, G. Di Paolo, S. Homma, P. A. Sims, V. K. Topkara and G. VunjakNovakovic, Nat. Biomed. Eng., 2018, 2, 293-303.

62 C. W. Chen, L. L. Wang, S. Zaman, J. Gordon, M. F. Arisi, C. M. Venkataraman, J. J. Chung, G. Hung, A. C. Gaffey, L. A. Spruce, H. Fazelinia, R. C. Gorman, S. H. Seeholzer, J. A. Burdick and P. Atluri, Cardiovasc. Res., 2018, 114, 1029-1040.

63 C. Han, J. Zhou, C. Liang, B. Liu, X. Pan, Y. Zhang, Y. Wang, B. Yan, W. Xie, F. Liu, X. Y. Yu and Y. Li, Biomater. Sci., 2019, 7, 2920-2933.

64 Y. Zhang, P. Zhang, X. Gao, L. Chang, Z. Chen and X. Mei, Mater. Sci. Eng., C, 2021, 120, 111671.

65 J. S. Chin, W. H. Chooi, H. Wang, W. Ong, K. W. Leong and S. Y. Chew, Acta Biomater., 2019, 90, 60-70.

66 C. Wang, C. Liang, R. Wang, X. Yao, P. Guo, W. Yuan, Y. Liu, Y. Song, Z. Li and X. Xie, Biomater. Sci., 2019, 8, 313-324.

67 Q. Shi, Z. Qian, D. Liu, J. Sun, X. Wang, H. Liu, J. Xu and X. Guo, Front. Physiol., 2017, 8, 904.
68 S.-C. Tao, S.-C. Guo, M. Li, Q.-F. Ke, Y.-P. Guo and C.-Q. Zhang, Stem Cells Transl. Med., 2017, 6, 736-747.

69 M. Li, Q. F. Ke, S. C. Tao, S. C. Guo, B. Y. Rui and Y. P. Guo, J. Mater. Chem. B, 2016, 4, 6830-6841.

70 S. Chen, Y. Tang, Y. Liu, P. Zhang, L. Lv, X. Zhang, L. Jia and Y. Zhou, Cell Proliferation, 2019, 52, e12669.

71 X. Liu, Y. Yang, Y. Li, X. Niu, B. Zhao and Y. Wang, Nanoscale, 2017, 9, 4430-4438.

72 K. Zhang, X. Zhao, X. Chen, Y. Wei, W. Du, Y. Wang, L. Liu, W. Zhao, Z. Han, D. Kong, Q. Zhao, Z. Guo, Z. Han, N. Liu, F. Ma and Z. Li, ACS Appl. Mater. Interfaces, 2018, 10, 30081-30091.

73 L. Xin, X. Lin, F. Zhou, C. Li, X. Wang, H. Yu, Y. Pan, H. Fei, L. Ma and S. Zhang, Acta Biomater., 2020, 113, 252-266.

74 N. Xu, L. Wang, J. Guan, C. Tang, N. He, W. Zhang and S. Fu, Int. J. Biol. Macromol., 2018, 117, 102-107.

75 S. Yang, B. Zhu, P. Yin, L. Zhao, Y. Wang, Z. Fu, R. Dang, J. Xu and J. Zhang, ACS Biomater. Sci. Eng., 2020, 6, 1590-1602.

76 W. Li, Y. Liu, P. Zhang, Y. Tang, M. Zhou, W. Jiang, X. Zhang, G. Wu and Y. Zhou, ACS Appl. Mater. Interfaces, 2018, 10, 5240-5254.

77 I. L. Colao, R. Corteling, D. Bracewell and I. Wall, Trends Mol. Med., 2018, 24, 242-256.

78 S. A. Kooijmans, P. Vader, S. M. van Dommelen, W. W. van Solinge and R. M. Schiffelers, Int. J. Nanomed., 2012, 7, 1525-1541.

79 J. Xu, Q. Feng, S. Lin, W. Yuan, R. Li, J. Li, K. Wei, X. Chen, K. Zhang, Y. Yang, T. Wu, B. Wang, M. Zhu, R. Guo, G. Li and L. Bian, Biomaterials, 2019, 210, 5161.

80 C. D. Pritchard, T. M. O'Shea, D. J. Siegwart, E. Calo, D. G. Anderson, F. M. Reynolds, J. A. Thomas, J. R. Slotkin, E. J. Woodard and R. Langer, Biomaterials, 2011, 32, 587597.

81 M. Liu, X. Zeng, C. Ma, H. Yi, Z. Ali, X. Mou, S. Li, Y. Deng and N. He, Bone Res., 2017, 5, 17014.

82 J. Huang, Z. Huang, Y. Liang, W. Yuan, L. Bian, L. Duan, Z. Rong, J. Xiong, D. Wang and J. Xia, Biomater. Sci., 2021, 9, 2602-2630.

83 Y. Liang, L. Duan, J. Lu and J. Xia, Theranostics, 2021, 11, 3183-3195.

84 L. Duan, L. Xu, X. Xu, Z. Qin, X. Zhou, Y. Xiao, Y. Liang and J. Xia, Nanoscale, 2021, 13, 1387-1397.

85 Y. Liang, X. Xu, X. Li, J. Xiong, B. Li, L. Duan, D. Wang and J. Xia, ACS Appl. Mater. Interfaces, 2020, 12, 3693836947.

86 X. Xu, Y. Liang, X. Li, K. Ouyang, M. Wang, T. Cao, W. Li, J. Liu, J. Xiong, B. Li, J. Xia, D. Wang and L. Duan, Biomaterials, 2021, 269, 120539. 